\title{
Globale und lokale Produktionsnetzwerke der balinesischen Bekleidungsindustrie
}

\author{
Markus Hassler, Bochum
}

\section{Einleitung}

Die Bekleidungs-, speziell die Batikindustrie, gehört zu den bedeutendsten Branchen innerhalb des verarbeitenden Gewerbes in Indonesien. Die räumliche Verteilung der Ansiedlung dieser Branche innerhalb Indonesiens konzentriert sich jedoch stark auf wenige Inseln dieses Archipels. Neben Java, dem ökonomischen Zentrum Indonesiens, haben nur Bali und Teile Sumatras eine Bedeutung als Standort für die industrielle Fertigung von Bekleidung (HASSLER 2004a). Bali hat dabei nach Java die zweithöchste Dichte an Bekleidungsproduzenten. Die hergestellten Produkte unterscheiden sich jedoch von denen, die primär in Java gefertigt werden. Während die export-orientierte Bekleidungsindustrie von Java vor allem Bekleidung für etablierte Marken und Käufer-Unternehmen aus den global bedeutenden Konsumgütermärkten Nordamerika und Westeuropa fertigt, haben sich balinesische Bekleidungsunternehmen vor allem auf die Herstellung von Bekleidung aus Batik und Stickarbeiten spezialisiert. Der Fokus auf diese beiden Produktgruppen ist vor allem im Kontext der steigenden Bedeutung des Tourismus auf dieser Insel und der damit gewachsenen Nachfrage nach Souvenirs zu sehen. Obwohl die Herstellung beider Produktgruppen eine lange Tradition in Indonesien hat - Batikbekleidung wurde ursprünglich in Mitteljava, vor allem in Surakarta, Yogyakarta und Kemalang hergestellt (GiLLow 1992; ReBECCA 1987), während Bekleidung mit Stickarbeiten (Kebaya) Teil der traditionellen Bekleidung Balis ist (RICHTER 1993) - entwickelte sich die balinesische Bekleidungsindustrie parallel zum Wachstum des balinesischen Tourismus (COLE 1998).

Die Dominanz dieser beiden Produkttypen hat zu besonderen Geschäftsnetzwerken innerhalb der balinesischen Bekleidungsindustrie geführt. Dies drückt sich sowohl in den Kooperationen mit den KäuferNetzwerken, als auch in der Organisation der Produktion aus. Obwohl die balinesische Bekleidungsindustrie ursprünglich vor allem lokale Souvenirmärkte bediente, liegt der Schwerpunkt heute maßgeblich auf einer Exportproduktion. Mehr als 90 Prozent aller auf Bali gefertigten Bekleidungstücke werden exportiert (Hassler 2004a). Die Organisation dieser ExportNetzwerke hängt dabei stark von der Herkunft und der Größe der Käufer-Unternehmen ab. Zudem ist im Gegensatz zu den globalen Strukturen der Vertriebs- organisation die Fertigung der Kleidungsstücke durch ein lokales Produktionsnetzwerk geprägt, wobei die Bekleidung vor allem von Heimarbeitern im ländlichen Raum Balis hergestellt wird.

Am Beispiel der Batikindustrie sollen diese spezifischen Strukturen der balinesischen Bekleidungsindustrie im Konzept der Warenketten untersucht werden. Daher werden zunächst einige wichtige Argumente der Debatte über Warenketten ausgearbeitet. Danach werden empirische Ergebnisse zur globalen Vernetzung der Vertriebsstrukturen und der lokalen Organisation der Heimarbeit dargestellt. Diese empirischen Ergebnisse beruhen auf zwanzig intensiven Experteninterviews, die mit Vertretern von Bekleidungsunternehmen in Bali durchgeführt wurden. Schließlich wird ein Fazit gezogen.

\section{Globale Produktionsnetzwerke und Warenketten}

Konzeptionelle Ansätze, die sich mit globalen Produktionsnetzwerken beschäftigen, basieren vor allem auf der Analyse einzelner Wirtschaftsakteure und deren Organisation in der Weltwirtschaft. Die Analyse konzentriert sich dabei vor allem auf die Art und Weise, wie Handel und Produktion über nationale Grenzen hinweg durchgeführt werden (vgl. Castells 2000; DiCKEN 2003). In diesem Kontext hat die Debatte über globale Warenketten einige besondere Argumente bezüglich der zunehmenden Internationalisierung wirtschaftlicher Aktivitäten entwickelt (vgl. Gereffi 1994, 1999, 2001). Zentraler Aspekt der Analyse innerhalb der Warenketten ist die länderübergreifende Segmentierung und Aufschlüsselung sämtlicher Wirtschaftsaktivitäten, die bei der Produktion und dem Konsum eines bestimmten Gutes von Bedeutung sind. Dies hat den Vorteil, dass einzelne Prozesse im gesamten Produktionssystem isoliert betrachtet werden können. Dabei werden die einzelnen Prozesse als «Knoten» im Netzwerkgeflecht der Warenkette betrachtet. Diese «Knoten» können klar bestimmt und auf ihre strukturellen Komponenten, welche verschiedene Wirtschaftsaktivitäten beinhalten wie zum Beispiel die Organisation der Rohwarenbeschaffung oder die Organisation von Transport, Vertrieb und Konsum (GerefFi et al. 1994), hin untersucht werden. Zudem kann das Verhältnis der eingesetzten Produktionsfaktoren betrachtet werden. Damit erlaubt das Konzept der Warenkette die Analyse der sozialen und organisatorischen Konstruktion und Zusammensetzung jeder Sequenz im gesamten Produktionsprozess (TAPLIN 
1994). Dies basiert auf dem Argument, dass innerhalb der gegenwärtigen globalen Wirtschaftsstruktur die «Knoten» den Ort der Profitgenerierung darstellen und nicht einzelne Nationalökonomien (APPELBAUM et al. 1994).

Die räumliche Ausdehnung dieser zentralen «Knoten» im Produktionsgeflecht definiert die Geographie oder Territorialität der Warenkette. Diese umfasst sowohl den tatsächliche Standort der «Knoten» als auch die räumliche Struktur der internen und externen Verflechtungen und Verbindungen der Unternehmen (APPElbaum et al. 1994). Daher weist die Räumlichkeit der Warenketten ein Spektrum von unterschiedlichen Ausdehnungen auf. Es reicht von räumlicher Dispersion bis zu hoher räumlichen Konzentration und von einer globalen Ausdehnung zu einer ausgesprochen lokalen Organisation (DICKEN 2003). Die räumliche Konfiguration der Warenkette ist dabei stark von Transport- und Kommunikationstechnologien beeinflusst. Die Innovationen innerhalb dieser Technologien, die eine organisatorische Integration räumlich disperser Wirtschaftsaktivitäten erlauben, haben zu einer kontinuierlichen räumlichen Ausdehnung von branchen-spezifischen Warenketten geführt.

Um die Formen der Integration von Unternehmen in die Weltwirtschaft zu identifizieren und die Machtverhältnisse zwischen den einzelnen Akteuren zu bestimmen, wurde in das Konzept der Warenketten die analytische Dimension der Governance-Struktur von Produktionsnetzwerken eingeführt. Die GovernanceStruktur jeder Warenkette ist stark von verschiedenen intra- und inter-organisatorischen Beziehungen abhängig. Im Konzept der Warenketten wird dabei primär zwischen produzenten-gesteuerten und käufer-gesteuerten Produktionssystemen unterschieden (GerefFi 1994, 1999, 2001). Diese Art der Produktionssteuerung ist vor allem das Ergebnis von unterschiedlichen Organisationsformen der führenden Unternehmen in der Weltwirtschaft. Bei der Definition von produzenten-gesteuerten Warenketten wird darauf hingewiesen, dass diese von Unternehmen koordiniert werden, die direkt in die Massenfertigung der Güter involviert sind. Ein wichtiger Faktor für die Wettbewerbsfähigkeit der Hauptunternehmen in diesen Produktionssystemen bildet dabei das produktionsspezifische Wissen. Als Beispiele für diese Organisationsstruktur werden Unternehmen aufgeführt, die in kapital- und technologieintensiven Industriebranchen tätig sind wie zum Beispiel die Automobil- oder die Flugzeugindustrie (vgl. GerefFI 1994, 1999).

Im Gegensatz dazu sind käufer-gesteuerte Warenketten dadurch gekennzeichnet, dass Hauptunternehmen über die Macht verfügen zu entscheiden wo, wann und wie die Produktion ausgeführt wird, ohne jedoch die Produktionsmittel direkt zu besitzen. $\mathrm{Zu}$ diesen Hauptunternehmen in käufer-gesteuerten Warenketten gehören große Handelsunternehmen und Markeneigentümer wie sie zum Beispiel in der Bekleidungs- oder Spielwarenindustrie tätig sind. Die Käufer-Unternehmen operieren mit einem Netzwerk externer. spezialisierter Produktionsunternehmen (RABACH \& KIM 1994). Das Hauptunternehmen lagert Teile oder die gesamte Produktion in spezialisierte Lohnunternehmen in verschiedenen Teilen der Erde aus (Gereffi 1995: 116). Diese Produktionsstruktur erlaubt es den Käufer-Unternehmen, sich auf die Veränderungen in den Konsummärkten zu konzentrieren und entsprechende strategische Entscheidungen zu treffen. Sie besitzen Marken und fördern diese durch Produktinnovationen oder neue Designs und die Entwicklung von zielgerichteten Marketingkampagnen in den Zielmärkten. Käufer-Unternehmen benutzen Marken als eine Quelle der Marktmacht (GEREFFI 2001: 33). Als primäres empirisches Beispiel zur Bestätigung dieser These der käufer-gesteuerten Warenkette wurde in verschiedenen Untersuchungen das Organisationsverhalten von Unternehmen in der Bekleidungsindustrie analysiert (vgl. z.B. BAIR \& GerefFI 2001; GerefFI 1994, 1999). GerefFi (1999: 40) sieht in den globalen Strukturen der Bekleidungsindustrie sogar den Prototypen einer käufer-gesteuerten Warenkette. Dabei beruht seine Aussage jedoch vor allem auf der Analyse von etablierten Markenunternehmen und Einzelhändlern in den global bedeutenden Hauptmärkten Nordamerika und Westeuropa.

Die konzeptionelle Entwicklung einer idealtypischen Organisationsstruktur innerhalb einer Industriebranche wurde aber verschiedentlich kritisiert. WHITLEY (1996: 419) argumentierte zum Beispiel, dass es stark vereinfachend sei, eine idealtypische Organisationsstruktur einer Industriebranche zu etablieren, zumal institutionelle Einflüsse wie z.B. politische und kulturelle Rahmenwerke oft auf das individuelle unternehmerische Verhalten einwirken. Der Dualismus zwischen produzenten-gesteuerten und käufer-gesteuerten Produktionssystemen im Konzept der Warenketten vereinfacht die potentiellen Variationen und Verschiedenartigkeiten der Machtverhältnisse und Verbindungen zwischen Unternehmen, die in der gleichen Industriebranche tätig sind (vgl. DickeN et al. 2001; SмIтH et al. 2002). Somit ist davon auszugehen, dass Bekleidungsindustrien, die andere Zielmärkte bedienen als die von GerefFI untersuchten globalen Hauptmärkte und dadurch anderen Machtstrukturen ausgesetzt sind oder mit spezifischen Produkten operieren wie zum Beispiel Batikbekleidung, in Warenketten eingebettet sind, die von der idealtypischen käufer-gesteuerten Warenkette abweichen. Daher sollen im Folgenden die Strukturen der Warenkette 
balinesische Batikindustrie untersucht und die besonderen Machtstrukturen darin hervorgehoben werden.

\section{Die Vertriebsstrukturen der balinesischen Bekleidungsindustrie}

Die Integration der balinesischen Bekleidungsindustrie in die Weltwirtschaft und die Organisation der Käufer-Unternehmen unterscheiden sich deutlich von denen der javanischen export-orientierten Bekleidungsindustrie. Obwohl die Käufer auch primär aus den globalen Hauptmärkten für Konsumgüter wie zum Beispiel den Ländern Nordamerikas, der Europäischen Union sowie Japan und Australien stammen (vgl.Hassler 2005a), sind die Unterschiede der Exportorganisation primär das Ergebnis der produzierten Bekleidung und der Größe der Käufer-Unternehmen. Die Abnehmer der balinesischen Bekleidung sind vor allem klein- und mittelständische Unternehmen, während die bedeutenden globalen Einzelhandelsketten und Markenunternehmen als Abnehmer keine Rolle spielen. Die einzelnen balinesischen Bekleidungsunternehmen operieren in der Regel mit einer grossen Anzahl von Kunden aus verschiedenen Ländern, doch ist eine Konzentration der Geschäftsaktivitäten auf einige Hauptabnehmer mit grossem Handelsvolumen gängig. Durch diese Hauptabnehmer werden bis zu 70 Prozent des Gesamtumsatzes erzielt. Zudem integrieren diese Käufer-Netzwerke oft intermediäre Agenten (Abbildung 1). Diese erfüllen vor allem bei der ersten Kontaktaufnahme zwischen Käufern aus Nordamerika und Westeuropa und dem balinesischen Hersteller wichtige Funktionen. Sie sind fast ausschließlich indonesischer Herkunft und haben in der Regel ihren Sitz in Jakarta. Die Integration ostasiatischer Agenten in die balinesischen Produktionsnetzwerke wie etwa bei javanischen export-orientierten Bekleidungsunternehmen (vgl. DiCKEn \& HASSLeR 2000; Hassler 2004b) ist nicht üblich. Abbildung 1 verdeutlicht die komplexe Vernetzung der verschiedenen Akteure und Produktionsbereiche im balinesischen Produktionssystem für Bekleidung.

Die Agenten begleiten ihre Kunden beziehungsweise die Einkäufer während ihrer Besuche nach Bali. Diese finden zwischen Juni und Oktober statt, welches die Hauptperiode ist, in der Käufer aus Nordamerika und Westeuropa ihre Bestellungen aufgeben. Die Rolle der Agenten verändert sich jedoch im Laufe der weiteren Entwicklung der Geschäftsnetzwerke. Dabei ist die Größe der Käufer-Unternehmen der wichtigste Einflussfaktor. Agenten, die in Netzwerken von gröBeren Käufern integriert sind, verbleiben in der Regel als wichtiger Bestandteil in der Warenkette. Sie übernehmen im weiteren Verlauf der Geschäftsaktivitäten Kontrollfunktionen über die Produktqualität und die termingerechte Lieferung. Viele kleine Käufer-Unter- nehmen versuchen jedoch, die Agenten aus ihren Netzwerken auszuschließen, um die direkten Kommissionskosten zu umgehen, welche durch die Dienstleistungen der Agenten entstehen. In diesem Fall werden die hergestellten Geschäftsbeziehungen aufrechterhalten und Folgebestellungen ohne die Hilfe von Agenten beim Hersteller aufgegeben. Der Mangel an Personal und die geringe Ausstattung mit Finanzmitteln führen in der Regel dazu, dass die Endkontrolle der Waren durch diese kleinen Käufer-Unternehmen nicht stattfindet. Vertrauen ist dabei das Hauptkriterium für diese Geschäftsbeziehungen, was sich auch in der Form der Bezahlung für die bestellten Kleidungsstücke ausdrückt. Bei diesen Transaktionen ist es üblich, 50 Prozent des Warenwertes vorab zu bezahlen und die restlichen 50 Prozent nach der Lieferung zu begleichen. Größere Käufer-Unternehmen benutzen Akkreditive als die gängige Form der Bezahlung bei internationalen Geschäften.

Die Größe der Käufer-Unternehmen hat ebenfalls einen entscheidenden Einfluß auf die Steuerung der Produktentwicklung. Die Kleinhändler sind zumeist nur Verkäufer von fertigen Kleidungsstücken, die sie in Bali einkaufen. Großhändler sind hingegen oft in der Lage, den Hersteller mit Produktspezifikationen zu versorgen, die aus einfachen Entwürfen und Skizzen der gewünschten Designs bestehen. Dagegen werden in der Regel auch von den besser organisierten Käufern keine genauen Schnittkonstruktionen der gewünschten Kleidungsstücke geliefert. Daher hat der balinesische Hersteller die Hauptverantwortung im Produktentwicklungsprozess für die neuen Kleidungsstücke. Dies steht im Gegensatz zur Definition der Organisation von Produktentwicklung in käufergesteuerten Produktionssystemen für Bekleidung nach Gereffi (1994, 1999). In dessen Definition werden sämtliche kreativen Prozesse von den Käufer-Unternehmen ausgeführt, wobei der Hersteller mit genauen Produktspezifikationen wie Designs, Schnittkonstruktionen und Grössentabellen beliefert wird. Der Hersteller ist ausschließlich mit Funktionen betraut, die der Ausführung der Massenfertigung dienen.

Der organisatorische Aufbau der Produktentwicklung in der balinesischen Batikfertigung ist neben dem schwachen Organisationsgrad der Käufer-Unternehmen durch die gefertigten Produkten begründet. Die Käufer verfügen nicht über die expliziten Kenntnisse der produktionsspezifischen Möglichkeiten und Grenzen der Batikfertigung. Daher fertigt das balinesische Unternehmen unabhängig von den spezifischen Angaben der internationalen Käufer eine komplette Musterkollektion, die den Einkäufern der Käufer-Unternehmen während ihrem Aufenthalt in Bali vorgelegt wird. In diesem Selektionsprozess ist es aber auch üblich, die vorbereiteten Muster nach den Wünschen der Käufer 
KÄUFER

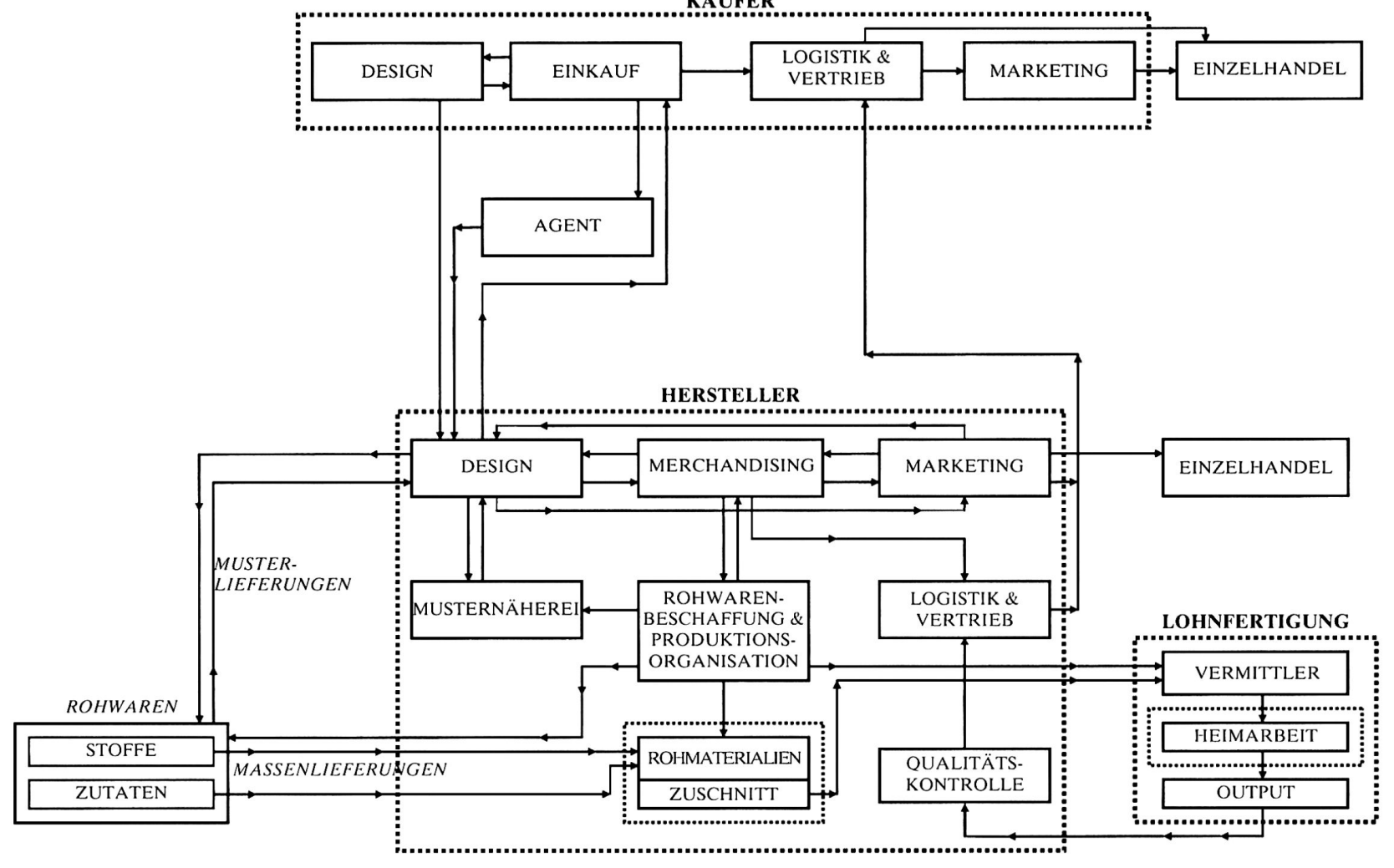

Abb. 1: Das Produktionssystem der balinesischen Bekleidungsindustrie

Production line of the Balinese clothing industry

Le système de production de l'industrie de l'habillement balinais 
zu verändern. Die Veränderung eines bestehenden Designs gilt als wesentlich einfacher und weniger zeitintensiv als der Entwurf und die Umsetzung eines komplett neuen Produkts.

\section{Die Organisation der Bekleidungsfertigung}

Während die Vertriebsaktivitäten der balinesischen Bekleidungsunternehmen vor allem eine globale Vernetzung aufweisen, ist die Fertigung der Kleidungsstücke von einer ausgesprochen lokalen Dimension geprägt (vgl. HASSLER 2005b). Die Näharbeiten werden vor allem von Heimarbeitern auf Bali durchgeführt. Somit ist das räumliche Muster der Batikherstellung dadurch gekennzeichnet, dass die Bekleidungsunternehmen, die das organisatorische Zentrum dieses Produktionssystems darstellen, ihren Standort vorwiegend in Denpasar haben, der Hauptstadt Balis, während die Heimarbeiter im ländlichen Hinterland Balis arbeiten. Zudem ist die Organisation der Produktion durch eine ausgeprägte räumliche Arbeitsteilung gekennzeichnet. Die Hauptakteure in diesem Produktionssystem sind dabei die Bekleidungsunternehmen als Auftraggeber, Arbeiter die an ihrem Wohnsitz arbeiten und Vermittler als verbindende Intermediäre (vgl. Abb. 1). Die Form der Kooperation dieser Akteure hat zu spezifischen Formen der Organisation und Arbeitsteilung geführt. Die Kommunikation zwischen Auftraggebern und Heimarbeitern findet dabei vor allem durch die Vermittler statt. Die Intermediäre sind gewöhnlich auch Teil einer Gruppe von Heimarbeitern und haben eine leitende Funktion. Sie erfüllen eine wichtige Rolle für beide Seiten des Produktionssystems, denn sie ermöglichen einen kontinuierlichen Informationsund Materialfluss zwischen Bekleidungsunternehmen und Heimarbeitern. Dies beinhaltet die Verteilung der zugeschnittenen Materialbündel, die Löhne der Heimarbeiter, das Einsammeln der gefertigten Waren und die Weitergabe an die Bekleidungsunternehmen.

Obwohl die Heimarbeiter externe Produktionskapazitäten für die Bekleidungsunternehmen darstellen, existieren sehr enge Geschäftsbeziehungen und Abhängigkeiten zwischen diesen Akteuren. Dies ist vor allem das Ergebnis der Bereitstellung des Finanzkapitals für die Beschaffung der Produktionsmittel der Heimarbeiter. Da alle Heimarbeiter ihre eigene Nähmaschine bereitstellen müssen, sind die Hürden zum Einstieg in diesen semi-industriellen Sektor relativ hoch. Die Bereitstellung und Verfügbarkeit der Finanzmittel, um die Produktionsmittel zu beschaffen und somit Beschäftigung und Arbeit ausserhalb landwirtschaftlicher Tätigkeiten zu finden, ist für die meisten ländlichen Haushalte sehr schwierig. Zudem ist der Zugang zu Bankkrediten für diese soziale Gruppe relativ schwer. Daher ist es üblich, dass die
Bekleidungsunternehmen den einzelnen Haushalten Darlehen für die Beschaffung von Nähmaschinen zur Verfügung stellen.

Diese finanzielle Unterstützung durch die Bekleidungsunternehmen ist ein wichtiger Faktor bei der Industrialisierung des ländlichen Raumes in Bali, da Beschäftigungsmöglichkeiten ausserhalb landwirtschaftlicher Tätigkeiten nur begrenzt vorhanden sind. Dabei haben diese Darlehen eine kurz- bis mittelfristige Laufzeit und werden monatlich von den Schuldnern zurückgezahlt. Obwohl diese engen finanziellen Verbindungen und Abhängigkeiten bestehen, wird von den Bekleidungsunternehmen keine exklusive Geschäftsbeziehung von ihren Kreditnehmern verlangt. Es wird nur eine vorrangige Behandlung in Zeiten der Hochsaison für die Produktion erwartet. Da das saisonale Muster der Produktion für die meisten balinesischen Bekleidungsunternehmen jedoch ähnlich verläuft, ist es ausgesprochen schwierig für die Heimarbeiter, in den Monaten geringer Nachfrage Arbeit und Aufträge zu finden. Da die meisten Käufer aus der nördlichen Hemisphäre kommen, entstehen bestimmte saisonale Muster bei der Nachfrage für die in Bali produzierte Sommerbekleidung. Die Zeiträume der geringen Nachfrage sind dabei Juni bis Mitte Oktober, während von Mitte Oktober bis April/ Mai eine hohe Nachfrage besteht.

Die von den Heimarbeitern eingesetzten Nähmaschinen haben einen sehr geringen technischen Standard und sind primär manuelle, mit Fußpedalen betriebene Nähmaschinen indonesischer Herkunft. Der geringe Preis im Vergleich zu Industrienähmaschinen ist dabei der Hauptvorteil dieser Produktionsmittel. Neben dieser grundlegenden Mechanisierung der Fertigung verlangt die Herstellung der Batikbekleidung aber auch in hohem Maße manuelle Arbeit. Das Flechten von Knoten bei der Herstellung von Batik-Sarongs oder das Annähen von Knöpfen aus unbehandelten, natürlichen Materialien wie Holz oder Muscheln sind ausschliesslich manuelle Tätigkeiten. Der Einsatz dieser Fertigungstätigkeiten oder dieser Materialien definiert zu einem grossen Teil die Besonderheiten der balinesischen Batikbekleidung. Somit bestimmen das Design der Kleidungstücke und die angewendeten Fertigungsprozesse zu einem großen Teil die Wettbewerbsfähigkeit der balinesischen Bekleidung. Der individuelle Charakter dieser Handarbeiten ist eine Besonderheit und unterstützt den Verkauf der fertigen Produkte. Dies drückt sich auch in der klaren Präferenz der Käufer für die manuell gefertigten Kleidungsstücke aus. So berichtete ein balinesischer Unternehmer davon, dass er eine Maschine für das Bedrucken der Stoffe mit Batikmustern gekauft habe. Damit sollten die Produktivität und die Perfektion der Produktion erhöht werden. Die Käufer waren jedoch mit der Gleichmäßigkeit und der 
Perfektion des Druckergebnisses nicht zufrieden und verlangten manuell bedruckte Stoffe. Das Argument der Käufer war, dass mit einem manuell bedruckten Stoff wesentlich höhere Preise erzielt würden, da ein handwerklich gefertigtes Erzeugnis ein individuelleres und somit hochwertigeres Erscheinungsbild habe. Dieses individuelle Aussehen der Stoffe und somit auch der Kleidungsstücke ist ein Hauptkriterium für den Kauf durch den Endverbraucher.

Die Tätigkeiten werden innerhalb externer Gruppen vorwiegend von Frauen in Heimarbeit im ländlichen Raum durchgeführt, eine gesellschaftliche Gruppe, die wenige Alternativen zur Beschäftigung und Arbeit ausserhalb der traditionellen Landwirtschaft hat. Die Herstellung von Souvenirs und Handarbeiten für ausländische Touristen, ebenfalls in Heimarbeit, bietet eine Alternative zur Bekleidungsfertigung. Eine der wenigen weiteren Möglichkeiten einer dauerhaften Beschäftigung bietet nur noch der Tourismussektor, was eine temporäre Migration bedeuten würde. Heimarbeit hat demgegenüber den Vorteil, dass die Beschäftigten in ihrer vertrauten Umgebung bleiben und häuslichen Tätigkeiten nachgehen können.

Die Tatsache, dass sehr wenige Beschäftigungsalternativen existieren, setzt die balinesischen Bekleidungsunternehmen in eine sehr vorteilhafte Position. Dadurch ist auch der Druck, die Arbeitsbedingungen zu verbessern, Sozialleistungen und Löhne über dem staatlich geregelten Minimum zu bezahlen, sehr gering. Die Arbeitnehmer bleiben in einer starken Abhängigkeit, und sie erhalten nur Lohn, wenn ihre Arbeit tatsächlich nachgefragt wird. Es gibt jedoch kein Einkommen, wenn die Arbeit nicht benötigt wird, auch nicht bei Krankheit oder anderen Gründen der Arbeitsunfähigkeit.

\section{Fazit}

Das Beispiel der balinesischen Bekleidungsindustrie verdeutlicht den Einfluss von Arbeitsbeziehungen auf die Strukturen von Warenketten. Die Tatsache, dass Arbeit und Produktionsmittel eine kombinierte und externe Organisation aufweisen, führt zu einer starken Machtposition der Bekleidungsunternehmen. Obwohl das Finanzkapital zur Beschaffung der Produktionsmittel von den Bekleidungsunternehmen zur Verfügung gestellt wird, muss dieses von den Heimarbeitern zurückbezahlt werden, was Abhängigkeiten von einem einzelnen Auftraggeber schafft. Diese unverbindlichen Produktions- und Arbeitsbeziehungen lassen Netzwerke mit starker Flexibilität entstehen und setzen die balineschen Bekleidungsunternehmen in das organisatorische Zentrum dieser Art der Warenkette. Diese Position wird zudem durch das produzierte Gut, hauptsächlich Batikbekleidung, gestärkt. Die Hauptinputs bei der Produktentwicklung für Batikmuster und Bekleidungsdesign werden durch diese Unternehmen generiert und/oder organisiert. Obwohl das internationale Käufer-Unternehmen die letzte Entscheidung über das Design des produzierten Kleidungsstücks trifft, bleibt das balinesische Unternehmen in einer Schlüsselposition innerhalb dieser Warenkette, da der Mehrwert in der Produktentwicklung in Bali geschaffen wird. Zudem wird die Wettbewerbsfähigkeit des Kleidungsstückes durch das Produkt und dessen besondere Charakteristika definiert und nicht durch den Markennamen des Käufer-Unternehmens.

Die Herstellerunternehmen haben eine dominante Marktposition gegenüber den internationalen Käufern, weil die Strukturen der Batikproduktion die Strukturen der Nachfrage gestalten. Dies kann auch als produzenten-gesteuerte Warenkette interpretiert werden. Da der Hauptmehrwert aber in den externen Produktionseinheiten von Heimarbeitern generiert wird, kann auch argumentiert werden, dass die Bekleidungsunternehmen Käufer repräsentieren. In der Produktionsstruktur der balinesischen Bekleidungsindustrie operieren die Unternehmen im zentralen «Knoten», während die Heimarbeiter eine periphere Rolle einnehmen. Das Beispiel der balinesischen Bekleidungsindustrie zeigt, dass die branchenspezifische duale Unterteilung der Governance-Struktur von Warenketten in GeREFFI's Modell zu wenig differenziert dargestellt ist. In bestimmten Branchen können Variationen in der Koordination von Warenketten entstehen, die sowohl durch das Produkt als auch durch institutionelle, standort-spezifische Faktoren beeinflusst werden können.

\section{Literatur}

Appelbaum, R.P., Smith, D. \& B. Christerson (1994): Commodity chains and industrial restructuring in the Pacific Rim: garment trade and manufacturing. - In: Gereffi, G. \& M. Korzeniewicz (Hrsg.): Commodity chains and global capitalism. - Westport, Conn., London: Greenwood Press: 187-204.

BaIR, J. \& G. GerefFi (2001): Local clusters in global chains: the causes and consequences of export dynamism in Torreon's blue jeans industry. - In: World development 29: 1885-1903.

Castells, M. (2000): The rise of the network society. $-2^{\text {nd }}$ edition, Oxford: Blackwell Publishers.

Cole, W. (1998): Bali's garment export industry. - In: Hill, H. \& K.W. Thee (Hrsg.): Indonesia's technological challenge. - Singapore: Institute of Southeast Asian Studies: 255-278.

Dicken, P. (2003): Global shift. Reshaping the global economic map in the $21^{\text {st }}$ century. - London: Guilford Press. 
Dicken, P. \& M. Hassler (2000): Organizing the Indonesian clothing industry in the global economy. The role of business networks. - In: Environment and planning A 32: 263-280.

Dicken, P., Kelly, P.F., Olds, K. \& H.W.-C. Yeung (2001): Chains and networks, territories and scales. Towards a relational framework for analysing the global economy. - In: Global networks 1: 89-112.

GerefFi, G. (1994): The organization of buyer-driven global commodity chains. How U.S. retailers shape overseas production networks. - In: GerefFI, G. \& M. KorZeniewicz (Hrsg.): Commodity chains and global capitalism. - Westport, Conn., London: Greenwood Press: 95-122.

GerefFI, G. (1995): Global production systems and third world development. - In: STallings, B. (Hrsg.): Global change, regional response. The new international context of development. - Cambridge, New York u.a.: Cambridge University Press: 100-142.

Gereffi, G. (1999): International trade and industrial upgrading in the apparel commodity chain. - In: Journal of international economics 48: 37-70.

GEREFFI, G. (2001): Beyond the producer-driven/buyerdriven dichotomy. The evolution of global value chains in the internet era. - In: Gereffi, G. \& R. Kaplinsky (Hrsg.): The value of value chains. Spreading the gains from globalisation, IDS Bulletin 32, 3: 30-40.

Gereffi, G., Korzeniewicz, M. \& R.P. Korzeniewicz (1994): Introduction: global commodity chains. - In: Gereffi, G. \& M. Korzeniewicz (Hrsg.): Commodity chains and global capitalism. - Westport, Conn., London: Greenwood Press: 1-14.

Gillow, J. (1992): Traditional Indonesian textiles. - London: Thames and Hudson.

HASSLER, M. (2004a): The geography of clothing production in Indonesia. Developments in time and space. - In: Asian geographer 23: 93-108.

HASSLER, M. (2004b): Raw material procurement, industrial upgrading and labor recruitment. Intermediaries in Indonesia's clothing industry. - In: Geoforum 35: 441-451.

HASSLER, M. (2005a): Global markets, local homeworking. Governance and inter-firm relationships in the Balinese clothing industry. - In: Geografiska annaler 87B: 31-43.

HASSLER, M. (2005b): Home-working in rural Bali. The organization of production and labor relations. - In: The professional geographer 57:530-538.

RABACH, E. \& E.M. KIM (1994): Where is the chain in commodity chains? The service sector nexus. - In: Gereffi, G. \& M. Korzeniewicz (Hrsg.): Commodity chains and global capitalism. - Westport, Conn., London: Greenwood Press: 123-142.

REBECCA, J. (1987): Worker, middlewomen, entrepreneur. Women in the Indonesian batik industry. - Bangkok: Population Council, Regional Office for South and East Asia.
Richter, A. (1993): Arts and crafts of Indonesia. London u.a.: Thames and Hudson.

Smith, A., Rainnie, A., Dunnford, M., Hardy, J., Hudson, R. \& D. Sadler (2002): Networks of value, commodities and regions. Reworking divisions of labour in macro-regional economies. - In: Progress in human geography 26: 41-63.

TAPLIN, I.M. (1994): Strategic reorientations of U.S. apparel firms. - In: GerefFi, G. \& M. Korzeniewicz (Hrsg.): Commodity chains and global capitalism. Westport, Conn., London: Greenwood Press: 205-222. WhitLey, R. (1996): Business systems and global commodity chains. Competing or complementary forms of economic organization. - In: Competition and change 1: 411-425.

\section{Zusammenfassung: Globale und lokale Produktions- netzwerke der balinesischen Bekleidungsindustrie}

Die zunehmende Vernetzung globaler Wirtschaftsaktivitäten hat zu einem Wachstum der Bekleidungsindustrien in den Entwicklungsländern geführt. Die globale Bekleidungsindustrie ist dabei durch Strukturen gekennzeichnet, die von einer internationalen Arbeitsteilung geprägt sind. Unternehmen in Industrienationen konzentrieren sich vor allem auf Produktentwicklung und Marketingaktivitäten, während in den Entwicklungsländern die Produktion durchgeführt wird. Diese Strukturen wurden in der Debatte zu globalen Warenketten als käufer-gesteuerte Produktionssysteme definiert. In diesem Kontext wird im vorliegenden Beitrag die balinesische Industrie für Batikbekleidung untersucht. Dies beinhaltet eine Untersuchung der Organisation sowohl der globalen Käufer-Netzwerke, als auch der lokalen Bekleidungsfertigung. Dabei wurde festgestellt, dass sich die Strukturen, in denen balinesische Batikhersteller operieren, sehr vom klassischen Muster der käufer-gesteuerten Warenkette unterscheiden.

\section{Summary: Global and local production networks of the Balinese clothing industry}

The increasing inter-connectivity of global economic activities has led to a strong growth of clothing industries in developing countries. This growth of the global clothing industry has developed within distinct structures, which are characterised by an international division of labour. Firms in developed countries concentrate predominantly on product development processes and marketing activities, while production is conducted in developing countries. These structures have been identified within the debate on global commodity chains as buyer-driven production systems. Within this context, this paper aims to investigate the Balinese clothing industry. This includes an investigation of the organisation of global buyer networks as well as of local clothing production. A main finding 
of this research has been, that the commodity chain structures of Balinese clothing firms are very different from those patterns identified in the classic buyerdriven chain.

\section{Résumé: Les réseaux globaux et locaux de production} de l'industrie de l'habillement balinaise

La mise en réseau accrue des activités économiques a entraîné une croissance de l'industrie de l'habillement dans les pays en voie de développement. L'industrie globale de l'habillement est ainsi caractérisée par une division internationale du travail. Dans les pays industriels, les entreprises sont concentrées sur le développement des produits et les activités de marketing, alors que la production est réalisée dans les pays en voie de développement. Ces structures ont été définies dans la littérature traitant les chaînes de marchandises globales comme des systèmes de production contrôlés par le client (buyer-driven). La présente contribution examine, dans ce contexte, l'industrie balinaise de l'habillement en batik, à travers une analyse de l'organi- sation des réseaux globaux des acheteurs ainsi que de la fabrication locale. Force est alors de constater que les structures dans lesquelles les producteurs balinais travaillent se différencient fortement du modèle classique de la chaîne de marchandises contrôlée par le client.

Dr. Markus Hassler, Geographisches Institut, RuhrUniversität Bochum, Universitätsstrasse 150, D-44780 Bochum.

e-mail: markus.hassler@ruhr-uni-bochum.de

Manuskripteingang/received/manuscrit entré le 3.11.2005

Annahme zum Druck/accepted for publication/accepté pour l'impression: 29.3.2006 